\title{
Modificación superficial de un acero AISI SAE 1045 mediante la implantación de iones de nitrógeno y titanio
}

\author{
Ely Dannier V. Niño \\ MSc en Física, Universidad Industrial de Santander \\ Docente Tiempo Completo, Investigador Grupo GINTEP, \\ Universidad Pontificia Bolivariana UPB, Investigador Grupo FITEK, \\ Universidad Industrial de Santander UIS \\ Bucaramanga, Colombia \\ ely.valbuena@upb.edu.co \\ Darío Y. Peña \\ PhD en Corrosión, Universidad de Manchester \\ Docente Tiempo Completo, Investigador Grupo GIC, \\ Universidad Industrial de Santander UIS \\ Bucaramanga, Colombia \\ dypena@uis.edu.co
}

\begin{abstract}
Resumen- Los constantes avances tecnológicos han permitido generar técnicas para la protección superficial de materiales expuestos en medios agresivos; por ello el Grupo de Investigación en Tecnología del Plasma y Corrosión (FITEK) junto con el Grupo de Investigación en Corrosión (GIC) de la Universidad Industrial de Santander (UIS), desarrollan una nueva tecnología de modificación superficial de sólidos mediante descargas pulsadas de alto voltaje y de arco eléctrico a bajas presiones realizada en el reactor JÚPITER (Joint Universal Plasma and Ion Technologies Experimental Reactor). La presente investigación buscó mediante ensayos de inspección visual, espectroscopia de plasma inducido por láser y microscopía óptica, evaluar el desempeño de las superficies del acero AISI SAE 1045 no modificadas y modificadas con iones de especies no metálicas (Nitrógeno) y metálicas (Titanio), en el reactor de Modificación Superficial de Metales (MOSMET) después de estar inmersas por varios días en solución de cloruro de sodio ( $\mathrm{NaCl}$ ) al $3 \%$. La dosis implantada a 9 minutos con titanio tuvo un mejor comportamiento en los ensayos de corrosión en solución $\mathrm{NaCl}$ al $3 \%$, estableció la implantación con iones de titanio, como un posible mecanismo efectivo para la protección contra el deterioro del acero AISI SAE 1045 expuesto a medios agresivos.
\end{abstract}

Palabras clave- Acero AISI SAE 1045, Modificación Superficial, Productos de Corrosión, Inspección Visual, Espectroscopia de Plasmas Inducidos por Láser (LIBS), Microscopia Óptica.

Abstract- Modern metal-mechanical industry requires new technologies to protect metal surfaces in aggressive media. To meet these needs the Research Group on Plasma Technology and Corrosion (FITEK) together with the Corrosion Research Group (ICG) of the Industrial University of Santander (UIS), are developing a new technology modify solids surface by high-voltage pulsed discharge and electric arc at low pressures in the JUPITER

\author{
Deicy Viviana Salinas \\ Ing Metalúrgica, Universidad Industrial de Santander \\ Investigador Grupo GIC, Universidad Industrial de Santander UIS, \\ Investigador Grupo GINTEP, \\ Universidad Pontificia Bolivariana UPB \\ Bucaramanga, Colombia \\ Luisa Fernanda Chinchilla \\ Ing Metalúrgica, Universidad Industrial de Santander \\ Investigador Grupo GIC, Universidad Industrial de Santander UIS, \\ Investigador Grupo GINTEP, \\ Universidad Pontificia Bolivariana UPB \\ Bucaramanga, Colombia
}

reactor (Joint Universal Plasma and Ion Technologies Experimental Reactor). Present investigation sought by testing visual inspection, spectroscopy, laser induced plasma and optical microscopy to evaluate the performance of the surfaces of SAE 1045 AISI unmodified and modified with metal ions species (nitrogen) and metal (titanium) in the reactor Surface Modification of Metals (MOSMET) after being immersed for several days in a solution of sodium chloride $(\mathrm{NaCl})$ to $3 \%$.The implanted dose to 9 minutes with titanium had a better performance in corrosion tests in $3 \% \mathrm{NaCl}$ solution, establishing the titanium ion implantation as a possible effective mechanism for protection against the erosion of AISI SAE 1045 steel exposed to aggressive media.

Keywords- AISI SAE 1045 steel, surface modification, corrosion products, visual inspection, spectroscopy, laser induced plasma, optical microscopy.

\section{INTRODUCCIÓN}

El mejoramiento de las propiedades superficiales de los materiales es una búsqueda continua y creciente de los distintos sectores industriales [1].

En relación con la corrosión, la modificación de las superficies comprende la aplicación de tecnologías para la obtención de propiedades deseadas que conllevan a la protección de diversos materiales sometidos a diferentes medios agresivos. Desde los métodos tradicionales como el esmaltado o la electrodeposición de metales, hasta las técnicas de desarrollo más reciente, como la implantación de iones y los tratamientos híbridos (Implantación - Deposición), son de interés mundial para mitigar los problemas de corrosión [2], [3]. 
En la década de los 70, se encontró que la implantación iónica en superficies metálicas podría mitigar los fenómenos de corrosión y aumentar la resistencia al desgaste; donde la capa implantada no es un enchape, no exige altas temperaturas de procesamiento, no aumenta el tamaño de los componentes y por no ser un proceso químico no produce contaminación ambiental [4].

Como una alternativa a los procesos de modificación superficial avanzada se presenta la implantación iónica tridimensional (Three Dimentional Ion Implantation - 3DII), la cual permite adecuar sin mayores costos sistemas que permiten aumentar significativamente la capacidad de funcionamiento de los equipos. A diferencia de las técnicas de implantación tradicional por haz de iones (IBI), Implantación de iones en fuentes de plasma (PSII) y la Implantación iónica por inmersión de Plasma; en la 3DII las probetas están inmersas en un plasma y toda la superficie es bombardeada simultáneamente por el flujo de iones sin necesidad de ser manipulada, lo que permite un tratamiento de alta calidad para piezas con formas irregulares [5].

De acuerdo a lo anterior, se analiza mediante Inspección Visual, Espectroscopia de Plasmas Inducidos por Láser (LIBS), Microscopia Óptica, el comportamiento de la resistencia a la corrosión en probetas de acero AISI SAE 1045 expuestas a un tratamiento superficial mediante descargas pulsadas de alto voltaje y de arco eléctrico a bajas presiones con iones de Nitrógeno y Titanio, en el reactor de Modificación Superficial de Metales (MOSMET) [6].

\section{METODOLOGÍA}

Se diseñaron y se elaboraron probetas (cupones), de acero AISI SAE 1045, con dimensiones $7.6 \mathrm{~cm} \times 1.9 \mathrm{~cm} \times 0.03 \mathrm{~cm}$, las cuales fueron preparadas superficialmente, por medio de limpieza mecánica y por ultrasonido [7]. Finalizada la limpieza superficial, se seleccionó un cupón de referencia para análisis metalográfico, el cual se llevó a cabo según la Norma ASTM E3-01[8].

Con la finalidad de eliminar la posible existencia de residuos sólidos y grasas superficiales antes de la modificación superficial [9], se realizó una limpieza por bombardeo iónico (sputtering a 5 $\mathrm{KV}$ ) con gas de argón en la cámara de descargas del reactor JÚPITER (ver Fig. 1).
La modificación superficial con iones de Ti y $\mathrm{N}$ se llevó a cabo mediante la técnica 3DII, implementada en el reactor JÚPITER; el proceso se desarrolló a partir de un flujo de átomos ionizados que incidían sobre la superficie del blanco metálico con una energía establecida (10 KeV) [10]. Las probetas fueron implantadas con Nitrógeno durante 60 y 90 minutos, y con Titanio durante 6 y 9 minutos.

FIG. 1. REACTOR JÚPITER

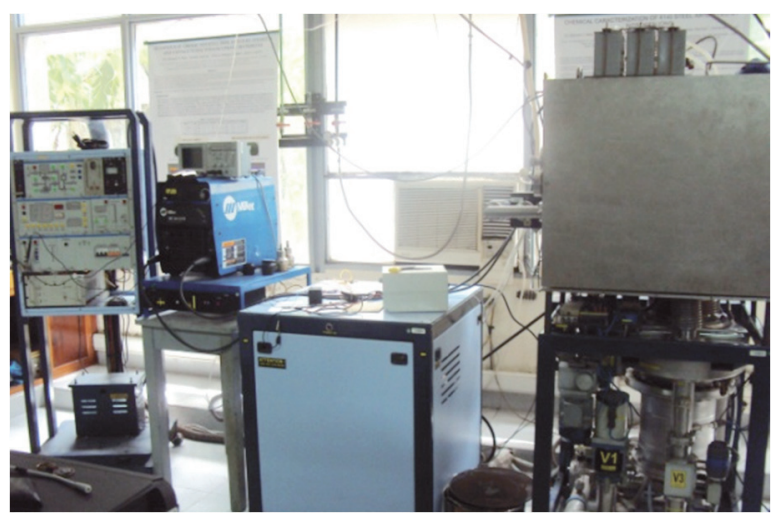

Fuente: Los autores

Posteriormente las superficies de los cupones implantados y no implantados estuvieron inmersas durante 28 días en una solución de cloruro de sodio $(\mathrm{NaCl})$ al 3\% [10].

Con el propósito de establecer la efectividad de la superficie modificada, por medio de la implantación de iones de nitrógeno y titanio, las superficies son caracterizadas antes y después de la inmersión en la solución de $\mathrm{NaCl}$ al 3\% mediante, Inspección Visual, Espectroscopia de Plasmas Inducidos por Láser (LIBS) y Microscopia Óptica [11]. El análisis multielemental de los productos de corrosión mediante la técnica LIBS, se realiza mediante un sistema instrumental con un espectrómetro Jarrell-Ash tipo Ebert ( $3.4 \mathrm{~m}$ de distancia focal) de alta resolución.

\section{RESULTADOS Y ANÁLISIS}

La inspección visual en las probetas implantadas y no implantadas se realizó de manera general donde se identificó la presencia de corrosión uniforme y corrosión por rendijas.

En la Fig. 2 y 3 se observan las superficies de las probetas implantadas y no implantadas después de 28 días de inmersión en solución de $\mathrm{NaCl}$ al 3\%. En las probetas implantadas con nitrógeno a 90 minutos y con titanio a 6 minutos, se obser- 
va corrosión localizada, encontrándose la posible presencia de óxidos como hematita (amarillo), hidróxidos (rojo-pardo) y cloruro férrico (azul-verdoso) [12], [13].

FIG. 2. SUPERFICIE DE REFERENCIA SIN MODIFICAR

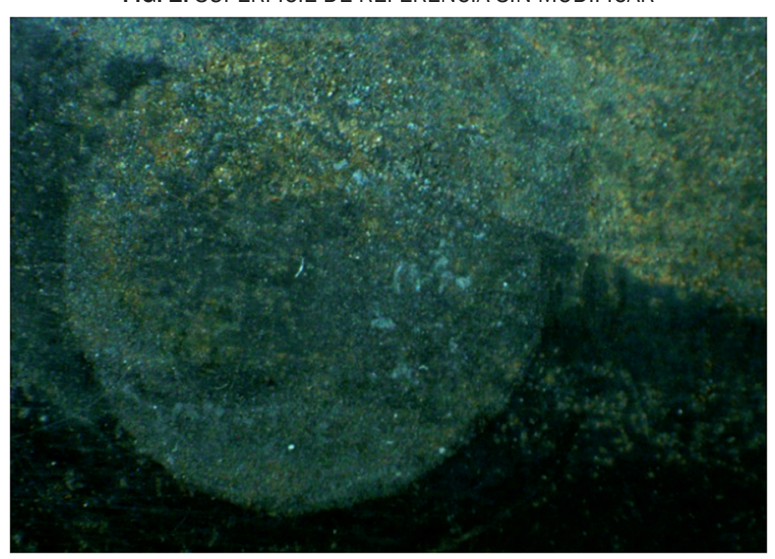

FIG. 3. SUPERFICIE IMPLANTADA CON (A) NITRÓGENO A 60 Y (B) 90 MINUTOS, (C) TITANIO A 6 Y (D) 9 MINUTOS

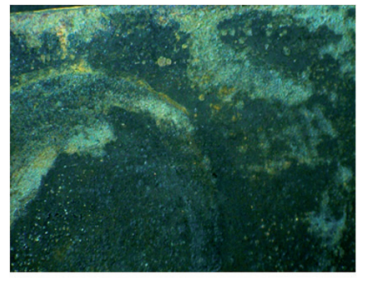

(A)

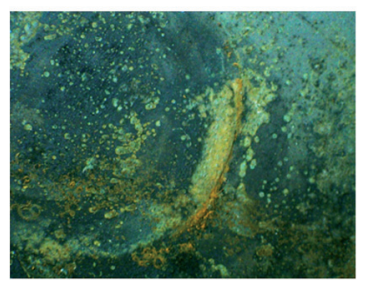

(C)

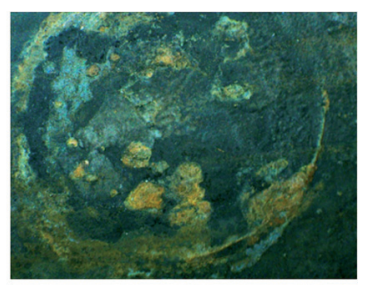

(B)

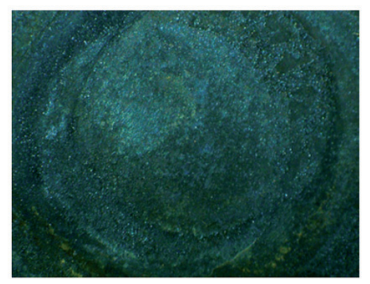

(D)
Fuente: Los autores

Mediante la técnica LIBS, se identificaron los elementos presentes en los productos de corrosión obtenidos de las probetas de acero AISI SAE 1045 implantadas con titanio y nitrógeno, después de estar inmersas en una solución de $\mathrm{NaCl}$ al $3 \%$.

En las Fig. 4(A) y 4(B) se muestran los espectros de intensidad respecto a la longitud de onda de los elementos presentes en los productos de corrosión de las probetas implantadas con titanio, tales como el Fe, Si, Ti, Na, entre otros [14], [15].
FIG. 4. ESPECTRO LIBS DE LOS PRODUCTOS DE CORROSIÓN EN PROBETAS IMPLANTADAS CON TITANIO (A) FE Y SI, (B) NA, TI Y FE

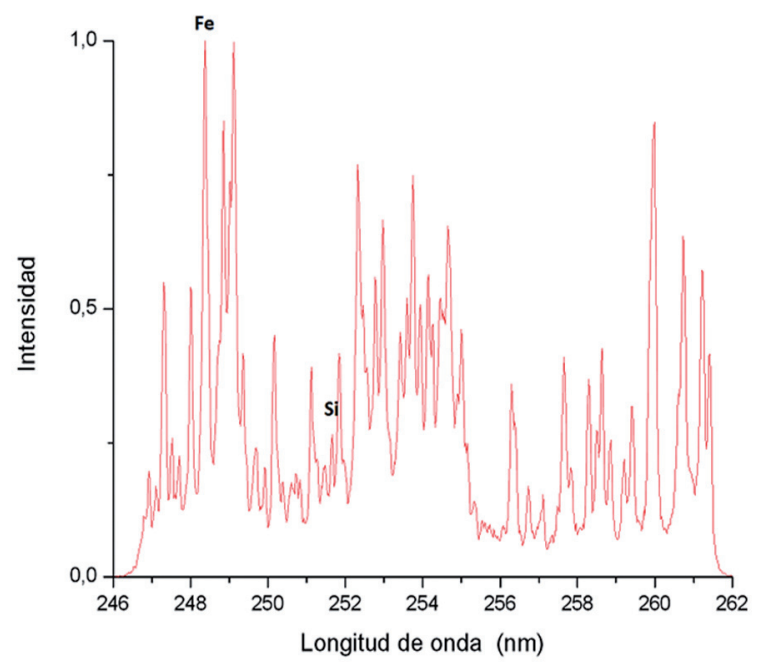

(A)

FIG. 4 (CONTINUACIÓN). ESPECTRO LIBS DE LOS PRODUCTOS DE CORROSIÓN EN PROBETAS IMPLANTADAS CON TITANIO (A) FE Y SI, (B) NA, TI Y FE

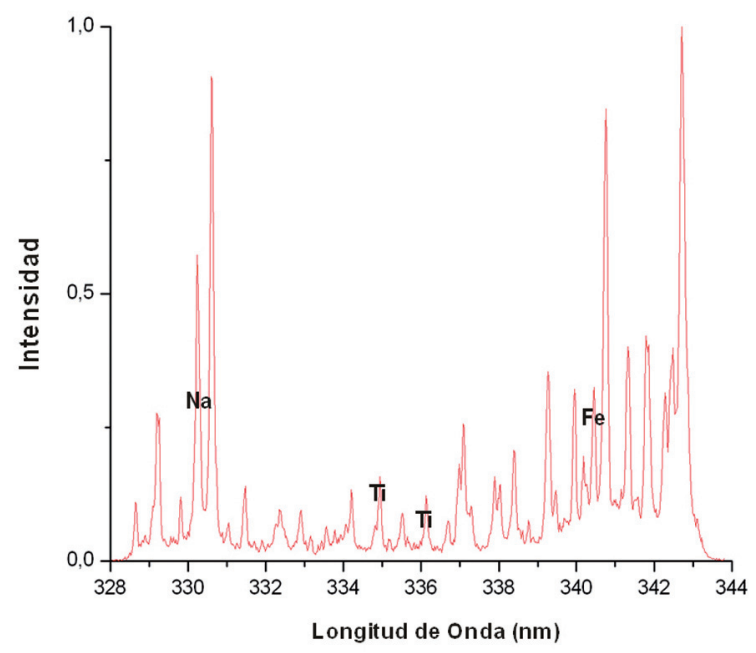

(B)

Fuente: Laboratorio LEAM - UIS

Los registros adquiridos para la textura y la topografía de la superficie en las muestras implantadas y no implantadas corresponden aproximadamente a la zona más afectada, donde se visualiza el mayor deterioro por picadura.

En las Fig. 5 ( $\mathrm{A}$ y B) se observó el mayor deterioro y formación de picaduras en la superficie de las muestras. En la Fig. 6 (A y B) se representa la topografía de la superficie observándose una rugosidad muy irregular. 
FIG. 5. TEXTURA DE LA PROBETA SIN IMPLANTAR (A) 0 (CERO) Y (B) 28 DÍAS DE INMERSIÓN

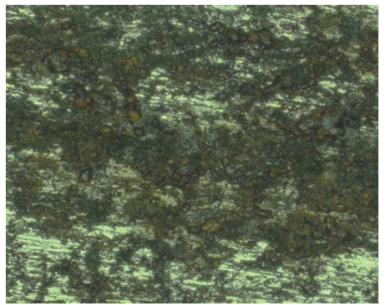

(A)

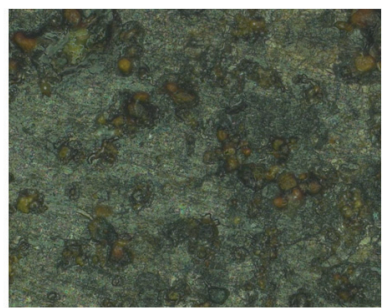

(B)

Fuente: GOTS - UIS

FIG. 6. TOPOGRAFÍA DE LA PROBETA SIN IMPLANTAR (A) 0 (CERO) Y (B) 28 DÍAS DE INMERSIÓN

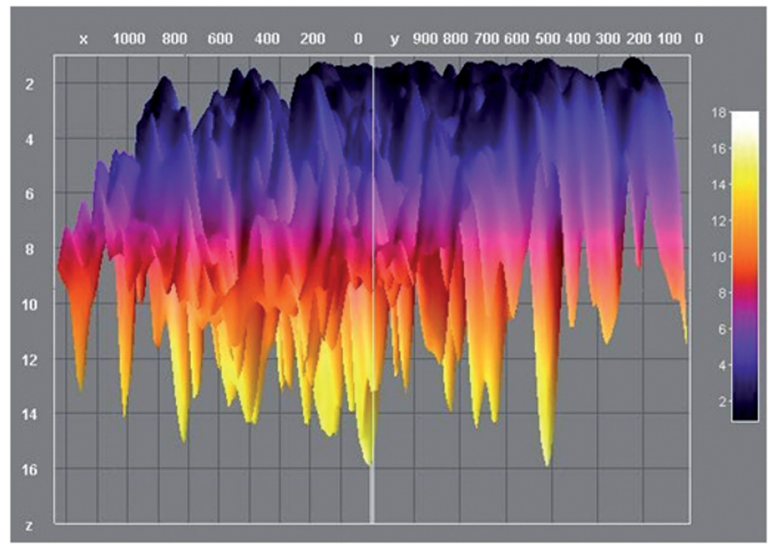

(A)

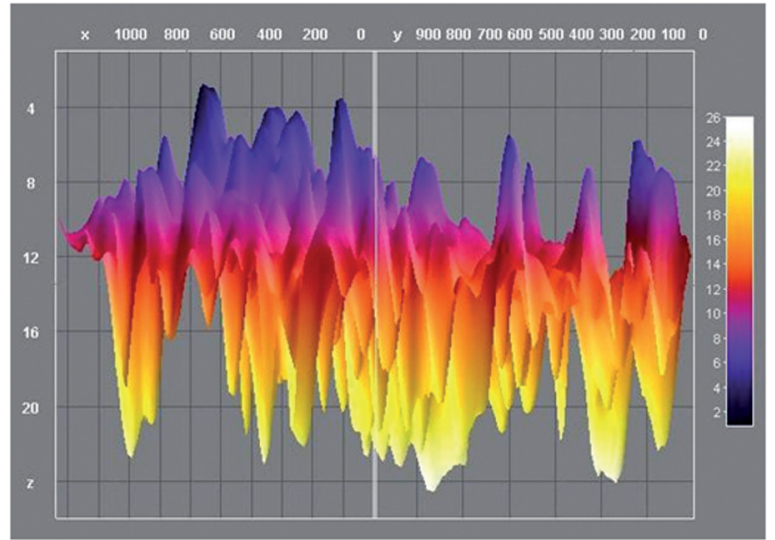

(B)

Fuente: GOTS - UIS

En las Fig. 7 (A) y 7 (B) se verifica la efectividad de la implantación de nitrógeno a un tiempo de 90 minutos, donde el deterioro de la superficie es relativamente menor comparada con la implantada con nitrógeno a un tiempo de 60 minutos. En la Fig. 8 (A) y 8 (B) la superficie de las probetas presenta un leve decapado que pudo ser causado por el bombardeo iónico durante la modificación superficial.

De igual forma, apreciamos un comportamiento más uniforme en la topografía de la probeta implantada con nitrógeno a 90 minutos que la implantada a 60 minutos.

FIG. 7. TEXTURA DE LA PROBETA IMPLANTADA CON NITRÓGENO (A) 60 Y (B) 90 MINUTOS

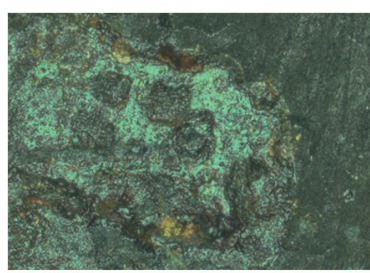

(A)

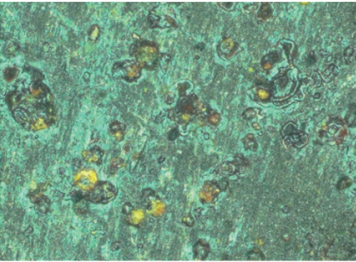

(B)
Fuente: GOTS - UIS

FIG. 8. TOPOGRAFÍA DE LA PROBETA IMPLANTADA CON NITRÓGENO (A) $60 \mathrm{Y}$ (B) 90 MINUTOS

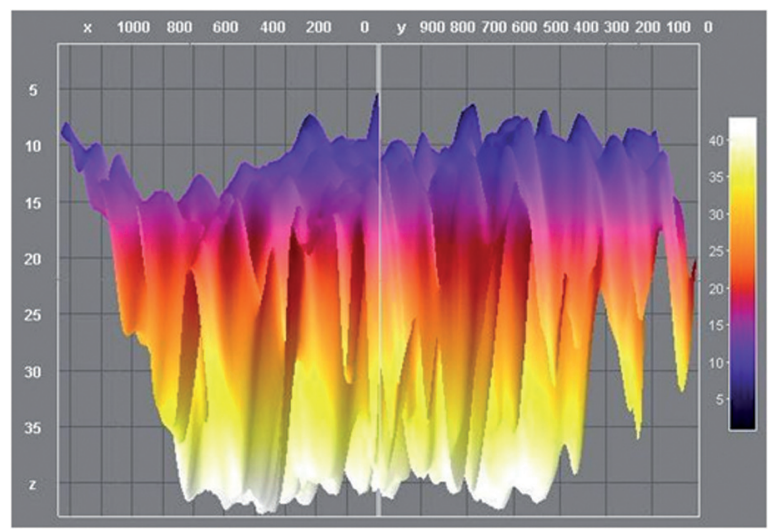

(A)

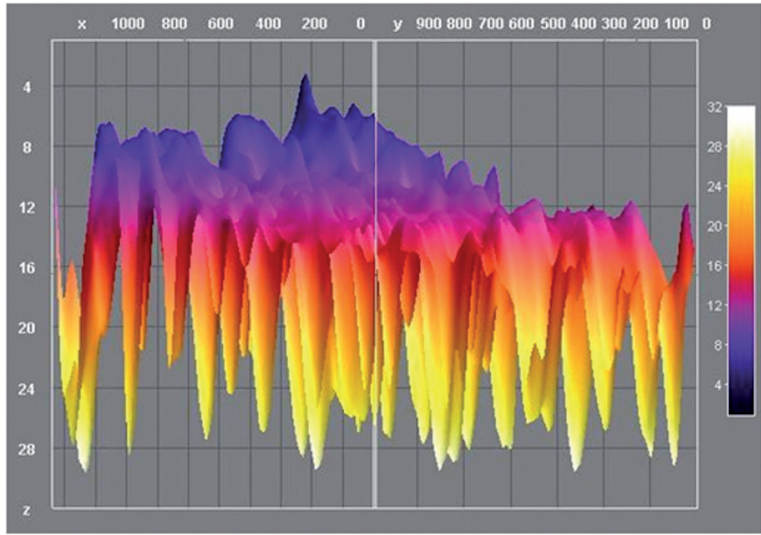

(B)

Fuente: GOTS - UIS

La Fig. 9 (A) y 9 (B) muestra la textura de las probetas implantadas con titanio a 6 y 9 minutos, donde se observa una disminución del deterioro super- 
ficial con respecto a las probetas no modificadas y modificadas con nitrógeno. En la Fig. 9 (A) se aprecia una región afectada (inferior derecha) por la corrosión por rendijas [16], donde se ve con mayor claridad en la Fig. 10 (A) (Zona de mayor protuberancia).

FIG. 9. TEXTURA DE LA PROBETA IMPLANTADA CON TITANIO (A) 6 Y (B) 9 MINUTOS

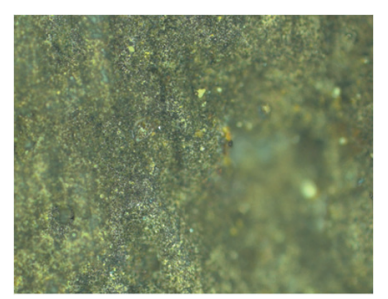

(A)

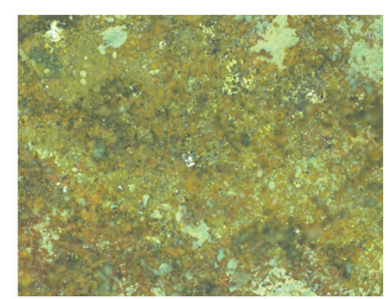

(B)
Fuente: GOTS - UIS

La topografía de las probetas implantadas con titanio a 6 y 9 minutos se muestra en la Fig. 10 (A y B). Al comparar las topografías de todas las muestras se observa que las probetas implantadas con titanio presentan una disminución muy significativa de la rugosidad, especialmente la tratada a 9 minutos de exposición.

De acuerdo con lo anterior se aprecia con facilidad que las superficies de las probetas implantadas e inmersas en la solución $\mathrm{NaCl}$ al $3 \%$ durante 28 días, presentaron una disminución de la rugosidad y menor degradación del material (formación de productos de corrosión, desgaste por picadura y rendija). Sin embargo, cabe resaltar el buen comportamiento encontrado en las probetas implantadas con titanio especialmente la modificada a 9 minutos.

FIG. 10. TOPOGRAFÍA DE LA PROBETA IMPLANTADA CON TITANIO (A) 6 Y (B) 9 MINUTOS

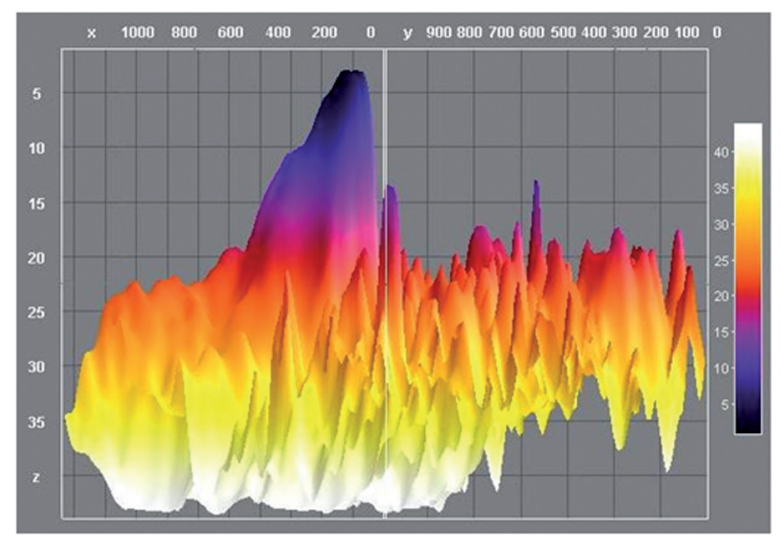

(A)

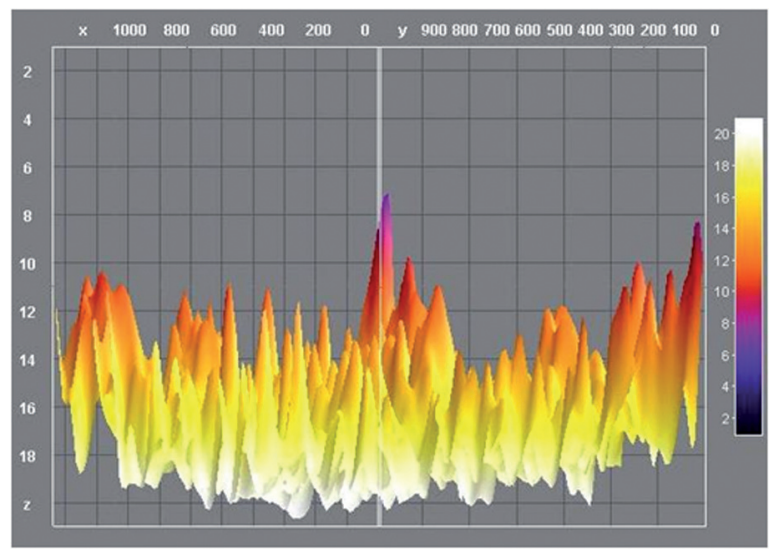

(B)

Fuente: GOTS - UIS

\section{CONCLUSIONES}

La implantación iónica tridimensional (3DII) realizada mediante descargas combinadas de arco eléctrico y pulsadas de alto voltaje a bajas presiones modifica superficialmente el acero AISI SAE 1045, y como consecuencia mitiga la corrosión en la superficie de la misma.

La dosis implantada a 9 minutos con titanio presentó el mejor comportamiento superficial después de la inmersión en solución $\mathrm{NaCl}$ al 3\%, que establece la implantación con iones de titanio, como un posible mecanismo efectivo para la protección contra el deterioro del acero AISI SAE 1045 expuesto a medios agresivos.

Los productos de corrosión formados en la superficie de las muestras tratadas y no tratadas con iones de nitrógeno y titanio expuestas a una solución de salmuera, se lograron identificar por medio de inspección visual, reconstrucción de topografía de superficies (microscopio de alta resolución) y por espectroscopia de plasmas inducidos por láser (LIBS).

\section{AGRADECIMIENTOS}

Los autores expresan sus agradecimientos a Colciencias por la financiación del Proyecto MOSMET, al Grupo de Investigación en Tecnología del Plasma y Corrosión (FITEK), al Grupo de Investigación en Corrosión (GIC), al Laboratorio de Espectroscopia Atómica y Molecular (LEAM), al Grupo de Óptica y Tratamiento de Señales (GOTS) de la Universidad Industrial de Santander y la Universidad Pontificia Bolivariana seccional Bucaramanga. 


\section{REFERENCIAS}

[1] RODRÍGUEZ, t. al (1998) "Presente y futuro de la implantación iónica": se describe la naturaleza, características, ventajas y desventajas de los tratamientos de superficie por implantación iónica; además el actual estado de desarrollo de esta tecnología, sus aplicaciones y las previsiones de su evolución en los próximos años.

[2] HUTCHINGS. r, (1994) A review of recent developments in ion implantation for metallurgical application: La implantación iónica surge como herramienta para mejorar la resistencia al desgaste y a la corrosión de los metales y aleaciones (materiales de ingeniería). Se realiza este trabajo o proyecto con el objetivo de identificar oportunidades para la aplicación industrial de la implantación iónica.

[3] MUTHUKUMARAN, V. Experimental investigation on corrosion and hardness of ion implanted AISI $316 \mathrm{~L}$ stainless steel. Materials \& desing technology. 2010

[4] SILVA, M. Estudio de la implantación iónica Tridimensional (3D II) en descargas a baja presión pulsadas como protección de aceros a la permeación de hidrógeno. Tesis de Magíster. Universidad Industrial de Santander, Bucaramanga, 2004

[5] DULCE Moreno, H. Jaime. Implantación lónica Tridimensional mediante descargas de alto voltaje a bajas presiones del dispositivo Júpiter. Tesis Doctoral, Universidad Industrial de Santander, Bucaramanga, 2002

[6] Proyecto Colciencias número 321 de 2005 Estudio de un Nuevo Método de Modificación de Superficies de Metales - MOSMET, Valeriy DugarZhabon.

[7] Fontalvo, Paola et al. Evaluación experimental de la resistencia a la corrosión de un Acero AISISAE 4140 Implantado con iones de Nitrógeno. En: Dyna. Colombia Vol. 76, No. 159, Septiembre 2009. p. 43-52

[8] ASTM E3-01 Standard Guide for Preparation of Metallographic Specimens

[9] RUEDA, A. comportamiento de la corrosión de un acero de bajo Carbono implantado con nitrógeno. En: Revista Colombina de Física, Vol. 38, No. 2, 2006. Alejandro Rueda V. et al. En: Revista coIombiana de física, Vol. 38, No. 2, 2006
[10] MUÑOZ, Castro Arturo. Tratamiento superficial de aceros Inoxidables por implantación iónica. Revista Contacto Nuclear. Departamento de Física. 4 p. Alejandro Rueda V. et al. En: Revista colombiana de física, Vol. 38, No. 2, 2006

[11] PEÑA, Darío et al. Evaluación Experimental de la Resistencia a la Corrosión de un Acero AISI-SAE 4140 Implantado con lones de Nitrógeno. En: Dyna. Medellín Vol.76, No.159, Sept. /Dic. 2009

[12] JONES, Denny A. Principles and Preventions of Corrosion. Prentice Hall: USA, 1996

[13] MIGUEL ÁNGEL BERMÚDEZ ODRIOSOLA, “Corrosión de las armaduras del hormigón armado en ambiente marino: zona de carrera de mareas y zona sumergida. Tesis Doctoral, Universidad, Universidad Politécnica de Madrid, Madrid, octubre del 2007".

[14] www.worldlingo.com/ma/enwiki/es/Laser/

[15] YÁÑEZ CASAL, Armando J, “Limpieza por láser como alternativa a los procesos de biorremediación en rocas". Laboratorio de Aplicaciones Industriales del Láser Centro de Investigación Tecnológica Universidad de Coruña., pp 4.

[16] VILA C., Gustavo. Electroquímica- Mecanismos y Métodos de Control. Universidad Nacional. 